\title{
Impact of local steroid application in a minimally invasive transforaminal lumbar interbody fusion: results of a prospective, randomized, single-blind trial
}

\author{
Brittany E. Haws, MD, Benjamin Khechen, BA, Dil V. Patel, BS, Mundeep S. Bawa, BA, \\ Junyoung Ahn, MD, Daniel D. Bohl, MD, MPH, Benjamin C. Mayo, MD, Dustin H. Massel, MD, \\ Jordan A. Guntin, BS, Kaitlyn L. Cardinal, BS, and Kern Singh, MD \\ Department of Orthopaedic Surgery, Rush University Medical Center, Chicago, Illinois
}

OBJECTIVE Local epidural steroid application may be associated with decreased pain and narcotic use in the immediate postoperative period following lumbar discectomy. However, local steroid delivery following lumbar fusion procedures has not been well characterized. This study aims to characterize the effect of local intraoperative depomedrol application on perioperative and postoperative outcomes following a single-level minimally invasive transforaminal lumbar interbody fusion (MIS TLIF).

METHODS A prospective, randomized, single-blinded study was performed. A priori power analysis determined that 86 patients were needed to detect a difference of 1 point in the visual analog scale (VAS) pain score between groups. Ninety-three patients were randomized into depomedrol (DEPO) and no depomedrol (NODEPO) cohorts. Prior to surgical closure, DEPO patients received $1 \mathrm{ml}$ depomedrol $(80 \mathrm{mg}$ ) applied directly to the surgical site by using a Gelfoam carrier. NODEPO patients received $1 \mathrm{ml}$ saline on the same Gelfoam carrier. Perioperative outcomes including acute postoperative pain and narcotic use were assessed for the duration of inpatient stay. Patient-reported outcomes (PROs) questionnaires including VAS back and leg pain scores, and Oswestry Disability Index (ODI) were administered preoperatively and at 6-week, 12-week, and 6-month follow-up. Outcomes for DEPO and NODEPO cohorts were compared using linear regression controlled for sex.

RESULTS Of the 93 patients, 45 (48.4\%) were randomized to DEPO and $48(51.6 \%)$ to NODEPO. A greater percentage of DEPO patients were female $(53.3 \%$ vs $27.1 \%, p=0.010)$. There were no other significant differences in patient baseline characteristics. Similarly, operating time, estimated blood loss, and length of inpatient stay did not differ between cohorts. Patients in the DEPO cohort consumed fewer hourly narcotics on postoperative day 0 (5.3 vs 6.3 oral morphine equivalents/hour, $p=0.034$ ). However, no differences in acute postoperative pain or total narcotics consumption were observed between groups. Preoperative VAS leg scores were statistically different between cohorts $(p=0.027)$. However, preoperative ODI and VAS back scores did not differ between groups. Additionally, DEPO and NODEPO groups experienced similar improvements in PROs at all postoperative time points.

CONCLUSIONS Local depomedrol use did not lead to decreases in acute postoperative pain or narcotics consumption after MIS TLIF. Additionally, local depomedrol was not associated with postoperative improvements in PROs. The findings of this randomized trial suggest that surgical and clinical outcomes following MIS TLIF may not be impacted by intraoperative application of depomedrol.

Clinical trial registration no.: NCT03308084 (clinicaltrials.gov)

https://thejns.org/doi/abs/10.3171/2018.7.SPINE18584

KEYWORDS minimally invasive transforaminal lumbar interbody fusion; depomedrol; postoperative pain; narcotics; intraoperative steroid

\footnotetext{
ABBREVIATIONS BMI = body mass index; $\mathrm{CCI}=$ Charlson Comorbidity Index; $\mathrm{DEPO}=$ depomedrol; LOS = length of hospital stay; MCID = minimum clinically important difference; MIS TLIF = minimally invasive transforaminal lumbar interbody fusion; NODEPO = no depomedrol; ODI = Oswestry Disability Index; OME = oral morphine equivalent; $P O D=$ postoperative day; PROs = patient-reported outcomes; VAS = visual analog scale.

SUBMITTED May 9, 2018. ACCEPTED July 26, 2018.
}

INCLUDE WHEN CITING Published online November 9, 2018; DOI: 10.3171/2018.7.SPINE18584. 
$\mathrm{P}$ OSTOPERATIVE pain is a common concern following lumbar spine surgery. Up to $40 \%$ of patients who undergo lumbar spine surgery experience recurrent or persistent postoperative pain that may develop into a prolonged hospital stay, chronic pain, and overuse of narcotic analgesics. ${ }^{11,13}$ Thus, efforts made to reduce postoperative pain may be advantageous in minimizing healthcare resource utilization and improving patient-reported outcomes (PROs).

Several studies have demonstrated reduced pain with the use of epidural steroids following lumbar spine surgery. Epidural steroids have demonstrated efficacy in decreasing postoperative Oswestry Disability Index (ODI) scores and reducing visual analog scale (VAS) back and leg pain scores in the early stages following lumbar discectomy. ${ }_{5}^{5,10,12,14}$ Additionally, epidural steroids have been associated with decreased length of hospital stay (LOS) and postoperative narcotic use in patients who undergo lumbar discectomy and laminectomy. ${ }^{13}$

However, few studies have investigated intraoperative local injection of corticosteroids at the surgical site in an effort to reduce the incidence and duration of postoperative pain after lumbar fusion procedures. In this context, this randomized controlled trial aims to determine the impact of local depomedrol application on perioperative and postoperative outcomes for patients undergoing a primary, single-level, minimally invasive transforaminal lumbar interbody fusion (MIS TLIF).

\section{Methods \\ Patient Population}

This study is registered at the US NIH (clinicaltrials. gov) \#NCT03308084. Following institutional review board approval, a prospective, randomized, single-blinded study was performed at a single academic medical center. Patients scheduled to undergo a primary, single-level MIS TLIF were considered for inclusion in the study. Patients were excluded if they had a history of allergic reaction or other contraindication to the medications used in the protocol, a medical history of gastrointestinal bleeding, or a history of lumbar spine trauma. Enrolled patients were randomized in depomedrol (DEPO) or no depomedrol (NODEPO) cohorts 1 day prior to the day of surgery by an investigator who was not involved in the patient's clinical care and who used a computerized random number generator. All patients were blinded to their treatment group assignment, but the senior surgeon was not. A total of 105 patients were enrolled between November 2015 and July $2017(\mathrm{DEPO}=52, \mathrm{NODEPO}=53)$. We hypothesized that the use of local intraoperative depomedrol would not have an impact on incidence or duration of postoperative pain, LOS, or clinical outcomes after MIS TLIF.

\section{Power Analysis}

A priori power analysis was performed based on a previous cohort of patients who underwent a 1-level MIS TLIF performed by the same surgeon. The average VAS pain score on postoperative day (POD) 1 in this population was $5.17 \pm 1.62$. A 1-point difference in the average VAS pain score between groups was set as the minimum needed for clinical relevance. Using a mean and SD of 5.17 \pm 1.62 for the control group, a power of $80 \%$, and alpha of 0.05 , it was determined that 86 patients were needed to detect a difference of 1 point in average VAS pain score between DEPO and NODEPO groups.

\section{Surgical Technique}

All MIS TLIF procedures were performed using a standard paramedian approach. ${ }^{9}$ Following endplate preparation, the interbody device was packed with local bone graft and either iliac crest bone graft or bone morphogenetic protein-2 and placed within the intervertebral space. Prior to surgical closure, DEPO patients received 1 $\mathrm{ml}$ of depomedrol $(80 \mathrm{mg})$ applied at the transforaminal space by using a $10-\mathrm{cm}^{2}$ Gelfoam carrier. NODEPO patients instead received $1 \mathrm{ml}$ of saline applied in the same manner. All patients received an intravenous dose of dexamethasone $(10 \mathrm{mg})$ at the beginning of the procedure. A multimodal analgesia protocol was used for standardized perioperative pain management between cohorts.

\section{Data Collection}

Patient baseline and perioperative characteristics were collected for each patient. Patient characteristics included age, sex, body mass index (BMI), smoking status, preoperative diagnosis, and comorbidity burden as measured by Charlson Comorbidity Index (CCI). A modified CCI with the age component removed was used to allow for the testing of comorbidity burden and age separately during statistical analysis. Perioperative variables including operating time, estimated intraoperative blood loss, postoperative LOS, and day of discharge were recorded. Any complications or reoperations during the perioperative or postoperative period were also recorded. Acute postoperative VAS pain scores during the inpatient period were recorded according to nursing protocols and averaged over each POD. Narcotics use for the duration of the inpatient stay was converted to oral morphine equivalents (OMEs), and then reported as a total and average per hour for each POD.

PROs questionnaires were administered preoperatively and at 6-week, 12-week, and 6-month postoperative time points. PRO measures included ODI, VAS back pain, and VAS leg pain scores. Achievement of minimum clinically important difference (MCID) in PROs at 6-month followup was determined using values proposed by Copay et al. ${ }^{4}$ MCID values for VAS back, VAS leg, and ODI were -1.2, -1.6 , and -12.8 , respectively.

\section{Statistical Analysis}

Statistical analysis was performed using Stata/MP 13.0 for Windows (StataCorp LP). Differences between DEPO and NODEPO cohorts in patient demographics and perioperative characteristics were assessed using independent t-tests for continuous variables and chi-square analysis for categorical variables. The association between local depomedrol use and inpatient pain or narcotics consumption was determined using linear regression controlled for sex. Improvements in PROs were compared between groups using linear regression controlled for sex. Differences in rates of MCID achievement between cohorts were tested 


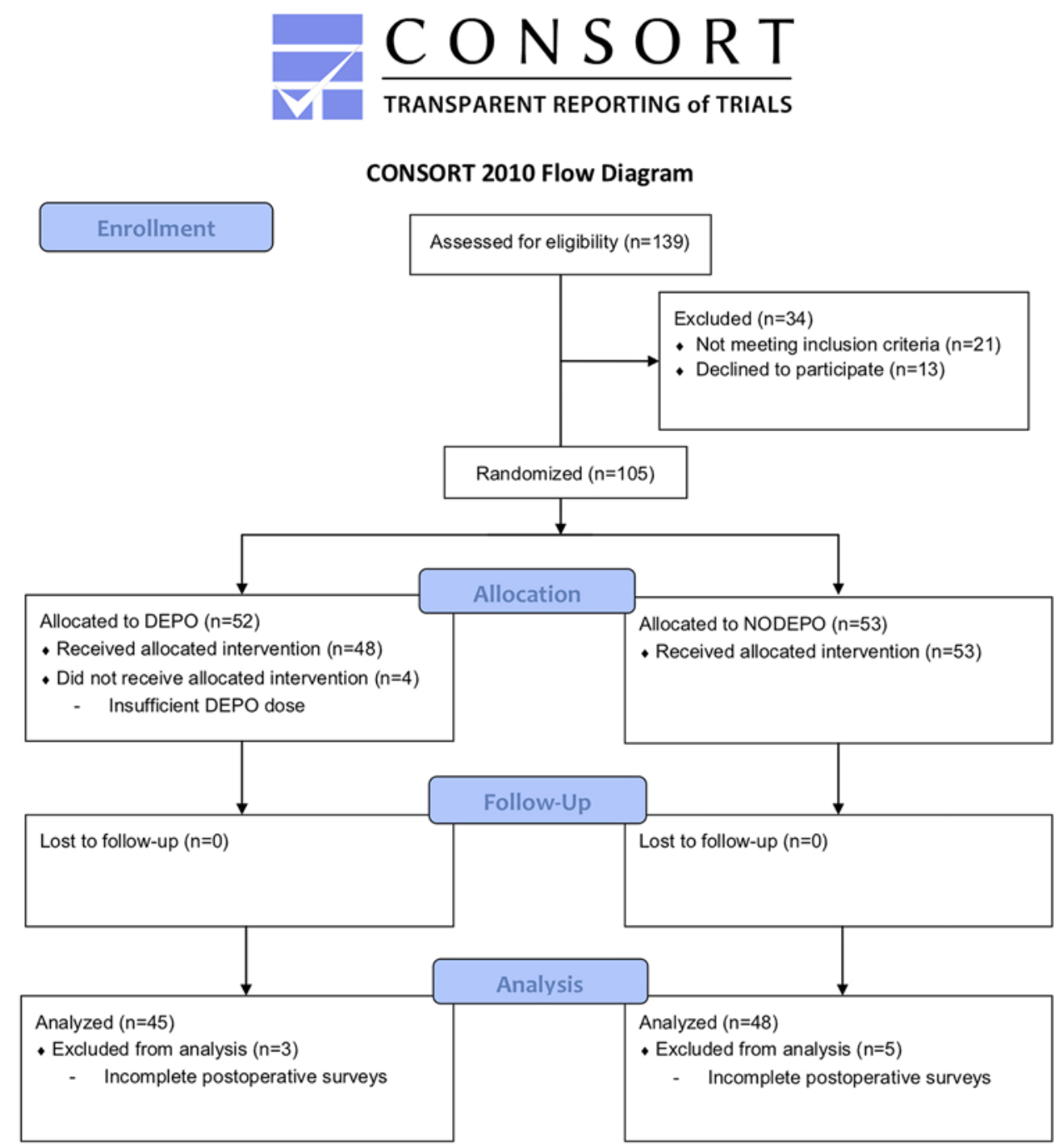

FIG. 1. Flow diagram describing the progress through each phase of the randomized controlled trial of the DEPO and NODEPO patient cohorts. Consolidated Standards of Reporting Trials (CONSORT) Flow Diagram template was downloaded from http:/l www.consort-statement.org/consort-statement/flow-diagram. Figure is available in color online only.

using Poisson regression with robust error variance controlled for sex. A p value of $<0.05$ was used to determine statistical significance.

\section{Results}

A total of 105 patients were enrolled and randomized to the DEPO $(\mathrm{n}=52)$ or NODEPO cohorts $(\mathrm{n}=53)$. Four patients in the DEPO cohort inadvertently received only a 40-mg injection of depomedrol, and therefore were excluded from analysis. An additional 8 patients were excluded from final analysis due to incomplete postoperative survey completion (3 in DEPO, 5 in NODEPO) (Fig. 1). Therefore, 93 patients were included in the final analysis, of which $45(48.4 \%)$ and $48(51.6 \%)$ were in DEPO and NODEPO groups, respectively. A greater percentage of DEPO patients were female $(53.3 \%$ vs $27.1 \%, \mathrm{p}=0.010)$. No significant differences in preoperative characteristics were identified between groups ( $p>0.05$; Table 1$)$.
Perioperative characteristics and complication rates are described in Table 2. Patients in the DEPO and NODEPO cohort exhibited similar operating times and intraoperative blood loss. The LOS and postoperative day of discharge were also found to be similar between groups. One patient in the DEPO cohort experienced postoperative urinary retention, requiring a urinary catheter upon discharge and follow-up with urology. In addition, 2 patients in the DEPO group developed superficial wound infections in the first 6 postoperative weeks that resolved with oral antibiotic therapy. Last, 1 patient in the DEPO cohort developed symptomatic pseudarthrosis, which required an anterior lumbar interbody fusion at the index level approximately 18 months postoperatively. No complications were observed in the NODEPO cohort. Statistically significant differences between groups for complication rates were not identified ( $p>0.05$ for each).

Inpatient pain scores and narcotics consumption are described in Table 3. No differences in acute postopera- 
TABLE 1. Baseline characteristics in 93 patients who underwent MIS TLIF

\begin{tabular}{|c|c|c|c|}
\hline Characteristic & $\begin{array}{c}\text { NODEPO, } \\
n=48\end{array}$ & $\begin{array}{l}\text { DEPO, } \\
n=45\end{array}$ & $\begin{array}{c}p \\
\text { Value* }\end{array}$ \\
\hline Age, yrs & $52.4 \pm 10.8$ & $51.8 \pm 11.2$ & 0.826 \\
\hline Sex & & & 0.010 \\
\hline Female & $27.1 \%(13)$ & $53.3 \%(24)$ & \\
\hline Male & $72.9 \%(35)$ & $46.7 \%(21)$ & \\
\hline BMI & & & 0.349 \\
\hline Nonobese, $\mathrm{BMI}<30$ & $45.8 \%(22)$ & $55.6 \%(25)$ & \\
\hline Obese, $\mathrm{BMI} \geq 30$ & $54.2 \%(26)$ & $44.4 \%(20)$ & \\
\hline Smoking status & & & 0.161 \\
\hline Nonsmoker & $91.7 \%(44)$ & $82.2 \%(37)$ & \\
\hline Smoker & $8.3 \%(4)$ & $17.8 \%(8)$ & \\
\hline Ageless comorbidity burden, $\mathrm{CCl}$ & $0.8 \pm 0.9$ & $1.1 \pm 1.1$ & 0.091 \\
\hline \multicolumn{4}{|l|}{ Preop diagnosis $†$} \\
\hline Degenerative spondylolisthesis & $62.5 \%(30)$ & $64.4 \%(29)$ & 0.846 \\
\hline Isthmic spondylolisthesis & $16.7 \%(8)$ & $13.3 \%(6)$ & 0.653 \\
\hline $\begin{array}{l}\text { Recurrent herniated nucleus } \\
\text { pulposus }\end{array}$ & $18.7 \%(9)$ & $20.0 \%(9)$ & 0.879 \\
\hline Degenerative disc disease & $54.2 \%(26)$ & $48.9 \%(22)$ & 0.611 \\
\hline Spinal stenosis & $93.8 \%(45)$ & $86.7 \%(39)$ & 0.248 \\
\hline
\end{tabular}

Boldface type indicates statistical significance. Values are expressed as the mean \pm SD or as the percentage (number of cases).

* $p$ values were calculated using chi-square analysis for categorical variables and independent t-test for continuous variables.

$\dagger$ Patients may have had multiple diagnoses.

tive VAS pain scores or total narcotics consumption were observed between DEPO and NODEPO groups $(\mathrm{p}>0.05)$. Patients in the DEPO cohort consumed fewer hourly narcotics on POD 0 (5.3 vs 6.3 OMEs/hour, $\mathrm{p}=0.034$ ). However, there were no differences between groups in hourly narcotics consumption on POD 1 or $2(\mathrm{p}>0.05)$.

Postoperative PRO improvements from preoperative scores are reported in Table 4. Preoperative VAS leg scores were statistically different between cohorts $(p=0.027)$. However, preoperative ODI and VAS back scores did not differ between groups $(p>0.05)$. Additionally, DEPO and NODEPO groups experienced similar improvements in PROs at all postoperative time points. Furthermore, patients in both cohorts achieved MCID for ODI, VAS back, and VAS leg at similar rates ( $\mathrm{p}>0.05$; Table 5).

\section{Discussion}

This randomized, controlled, single-blind trial investigated the effect of local intraoperative steroid application on perioperative and postoperative clinical outcomes in patients who underwent MIS TLIF. Although patients in the DEPO group on average consumed fewer narcotics per hour on POD 0, total narcotics consumption was not different between groups. The use of DEPO was not associated with reductions in LOS or acute postoperative pain. Additionally, patients in the DEPO cohort experienced similar complication rates and PROs as did those in the NODEPO group. These results suggest that the admin-
TABLE 2. Operative characteristics and complications in 93 patients who underwent MIS TLIF

\begin{tabular}{lccc}
\hline \multicolumn{1}{c}{ Characteristic } & $\begin{array}{c}\text { NODEPO, } \\
\mathrm{n}=48\end{array}$ & $\begin{array}{c}\text { DEPO, } \\
\mathrm{n}=45\end{array}$ & $\begin{array}{c}\mathrm{p} \\
\text { Value* }\end{array}$ \\
\hline Op time, mins & $112.6 \pm 24.2$ & $111.2 \pm 29.8$ & 0.806 \\
\hline Estimated blood loss, $\mathrm{ml}$ & $60.8 \pm 69.7$ & $61.3 \pm 71.9$ & 0.973 \\
\hline LOS, hrs & $32.3 \pm 23.9$ & $32.4 \pm 14.4$ & 0.979 \\
\hline Discharge day & & & 0.196 \\
\hline POD 0 & $18.7 \%(9)$ & $6.7 \%(3)$ & \\
\hline POD 1 & $56.3 \%(27)$ & $71.1 \%(32)$ & \\
\hline POD 2 & $14.6 \%(7)$ & $17.8 \%(8)$ & \\
\hline POD 3+ & $10.4 \%(5)$ & $4.4 \%(2)$ & \\
\hline Complications & & & \\
\hline Postop urinary retention & $0.0 \%(0)$ & $2.2 \%(1)$ & 0.299 \\
\hline Superficial wound infection & $0.0 \%(0)$ & $4.4 \%(2)$ & 0.140 \\
\hline Reops & $0.0 \%(0)$ & $2.2 \%(1)$ & 0.299 \\
\hline
\end{tabular}

Values are expressed as the mean $\pm \mathrm{SD}$ or as the percentage (number of cases).

${ }^{*} p$ values were calculated using chi-square analysis for categorical variables and independent t-test for continuous variables.

† Patient underwent anterior lumbar interbody fusion at index level 18 months postoperatively for symptomatic pseudarthrosis.

istration of local intraoperative steroids does not provide additional benefits with regard to surgical or clinical outcomes after MIS TLIF.

The similar acute postoperative pain and narcotics use between DEPO and NODEPO cohorts is in contrast to previous reports in the spine literature. Akinduro et al. performed a meta-analysis of 17 studies on the association between intraoperative epidural steroid use and outcomes in lumbar discectomy. ${ }^{1}$ Of the 12 studies reporting acute postoperative pain, $8(66.7 \%)$ indicated that steroid use was associated with significantly decreased pain. Additionally, 10 of 11 (90.9\%) studies reporting narcotic use demonstrated a decreased consumption among patients receiving steroids. These results have been supported by additional systematic reviews in the literature. 7,11

Although the majority of studies on local intraoperative steroids have been published in the lumbar decompression literature, the utility of steroids in lumbar fusion populations has not been thoroughly investigated. Jirarattanaphochai et al. performed a randomized, double-blind, controlled trial in 103 patients undergoing lumbar discectomy, laminectomy, and/or fusion. ${ }^{8}$ Patients were randomized to receive either methylprednisolone and bupivacaine or a saline injection applied to the surgical site prior to closure. Postoperative pain at rest was reported to be significantly lower in the methylprednisolone-bupivacaine group than in the control group (mean difference $-4.58, \mathrm{p}$ $=0.001$ ). Additionally, the cumulative morphine dose during the first 48 postoperative hours was significantly lower in the treatment group than in the placebo group (mean difference $-8.24 \mathrm{mg}, \mathrm{p}=0.01$ ). However, when stratifying by procedure type, no difference in morphine use was identified between groups for patients who underwent a lumbar fusion $(p=0.06)$. These results may suggest that local steroid application may not afford the same benefits 
TABLE 3. Inpatient pain scores and narcotics consumption in 93 patients who underwent MIS TLIF

\begin{tabular}{cccc}
\hline Factor & $\begin{array}{c}\text { NODEPO, } \\
n=48\end{array}$ & $\begin{array}{c}\text { DEPO, } \\
n=45\end{array}$ & $\begin{array}{c}p \\
\text { Value* }\end{array}$ \\
\hline Inpatient VAS pain scores & & & \\
\hline POD 0 & $5.5 \pm 1.8$ & $5.0 \pm 2.0$ & 0.089 \\
\hline POD 1 & $4.7 \pm 1.6$ & $4.5 \pm 2.0$ & 0.542 \\
\hline POD 2 & $5.6 \pm 1.5$ & $6.1 \pm 1.7$ & 0.950 \\
\hline Total daily OME consumption & & & \\
\hline POD 0 & $62.4 \pm 20.2$ & $59.5 \pm 22.3$ & 0.251 \\
\hline POD 1 & $58.5 \pm 26.0$ & $52.9 \pm 25.1$ & 0.360 \\
\hline POD 2 & $50.6 \pm 16.2$ & $49.3 \pm 28.2$ & 0.431 \\
\hline Hourly OME consumption & & & \\
\hline POD 0 & $6.3 \pm 2.5$ & $5.3 \pm 2.0$ & 0.034 \\
\hline POD 1 & $3.4 \pm 1.5$ & $3.0 \pm 1.1$ & 0.084 \\
\hline POD 2 & $3.3 \pm 1.8$ & $3.0 \pm 1.4$ & 0.134 \\
\hline
\end{tabular}

Boldface type indicates statistical significance. Values are expressed as the mean \pm SD.

${ }^{*} p$ values were calculated using linear regression controlled for sex.

in decreasing acute pain or narcotics use for more invasive procedures such as lumbar fusions.

Complications related to the administration of local intraoperative steroids remain a consideration with its routine use. In the present study a greater number of complications occurred in the DEPO cohort. These complications included 1 patient with postoperative urinary retention, 2 patients with superficial wound infections, and 1 patient requiring a repeat operation for symptomatic pseudarthrosis. However, this was not a statistically significant association. The aforementioned study by Akinduro et al. also investigated complication rates with intraoperative steroid use. ${ }^{1}$ In a meta-analysis, the authors identified a trend toward higher infectious $(0.94 \%$ vs $0.08 \%, \mathrm{p}=0.10)$ and total complication rate $(2.69 \%$ vs $1.18 \%, \mathrm{p}=0.19)$ among those receiving intraoperative steroids, although these were not statistically significant. It was suggested that this could be due to a low overall complication rate associated with lumbar discectomy that prevented statistically significant differences. These results, in combination with those of the present study, indicate the need for further investigation to better characterize the relationship between intraoperative steroid use and complication rates for MIS TLIF. Nevertheless, because the current literature is inconclusive, it would be prudent for surgeons to assess the potential risk for complications when considering the use of local intraoperative steroids for MIS TLIF.

In the present study, DEPO use did not lead to differences in patient-reported pain or disability up to 6 months postoperatively. Variable results regarding the association between steroid use and postoperative PROs have been reported in the literature. Ranguis et al. performed a systematic review of 12 randomized controlled trials to evaluate the efficacy of epidural steroids in lumbar spine surgery. ${ }^{11}$ In their meta-analysis, steroid use was associated with decreased radicular pain at 1-2 months postoperatively (mean difference $-2.14, \mathrm{p}=0.002$ ). However, no differ-
TABLE 4. Change in PROs in 93 patients who underwent MIS TLIF

\begin{tabular}{cccc}
\hline PROs & NODEPO, $\mathrm{n}=48$ & DEPO, $\mathrm{n}=45$ & $\mathrm{p} \mathrm{Value}{ }^{*}$ \\
\hline VAS back pain & & & \\
\hline Preop & $6.5 \pm 2.5$ & $6.4 \pm 2.6$ & 0.999 \\
\hline 6-wk $\Delta$ & $-2.5 \pm 2.8$ & $-3.0 \pm 2.9$ & 0.399 \\
\hline 12-wk $\Delta$ & $-2.7 \pm 2.9$ & $-2.9 \pm 3.1$ & 0.807 \\
\hline 6-mo $\Delta$ & $-3.6 \pm 3.3$ & $-2.9 \pm 3.3$ & 0.317 \\
\hline VAS leg pain & & & \\
\hline Preop & $6.5 \pm 2.6$ & $5.4 \pm 3.1$ & 0.027 \\
\hline 6-wk $\Delta$ & $-3.4 \pm 2.7$ & $-3.1 \pm 3.0$ & 0.588 \\
\hline 12-wk $\Delta$ & $-3.7 \pm 2.6$ & $-3.5 \pm 3.2$ & 0.518 \\
\hline 6-mo $\Delta$ & $-4.3 \pm 2.8$ & $-3.3 \pm 3.9$ & 0.079 \\
\hline ODI & & & \\
\hline Preop & $44.8 \pm 17.2$ & $40.8 \pm 16.4$ & 0.158 \\
\hline 6-wk $\Delta$ & $-8.5 \pm 15.0$ & $-6.5 \pm 20.4$ & 0.550 \\
\hline 12-wk $\Delta$ & $-11.4 \pm 17.6$ & $-13.6 \pm 16.0$ & 0.810 \\
\hline 6-mo $\Delta$ & $-20.9 \pm 20.2$ & $-18.7 \pm 17.3$ & 0.422 \\
\hline$\Delta$
\end{tabular}

$\Delta=$ change in value.

Boldface type indicates statistical significance. Values are expressed as the mean \pm SD.

${ }^{*} p$ values were calculated using linear regression controlled for sex.

ences in back pain at 1-2 months postoperatively were identified between treatment and control groups. Jirarattanaphochai et al. also investigated postoperative pain and ODI scores among patients undergoing lumbar spine surgery with either local intraoperative steroids or a placebo. ${ }^{8}$ Although back pain, leg pain, and ODI scores at 3-month follow-up were reported to be lower in the steroid group, this did not reach statistical significance.

No significant differences between DEPO and NODEPO groups were identified for a majority of outcomes assessed in the present study. The variability between these results and those within the spine literature may in part be due to publication bias. In the meta-analysis by Ranguis et al., a forest plot was created to assess the potential for publication bias. ${ }^{11}$ The authors suggested a high likelihood of publication bias or selective outcome reporting within their investigation. Enhanced transparency in reporting, particularly for negative or nonsignificant results, is encouraged in order to improve the evidence regarding the efficacy of intraoperative steroid use.

Our study has several limitations. First, all patients were treated by a single surgeon at a single institution, which may limit the study's generalizability. Second, al-

\section{TABLE 5. Percent of patients who achieved MCID}

\begin{tabular}{lccc}
\hline Pain Score & NODEPO, $n=48$ & DEPO, $n=45$ & p Value $^{*}$ \\
\hline ODI & $62.5 \%(30)$ & $55.6 \%(25)$ & 0.413 \\
\hline VAS back & $72.9 \%(35)$ & $62.2 \%(28)$ & 0.280 \\
\hline VAS leg & $72.9 \%(35)$ & $60.0 \%(27)$ & 0.111 \\
\hline
\end{tabular}

Values are expressed as the percentage (number of patients).

${ }^{*} p$ values were calculated using Poisson regression with robust error variance controlled for sex. 
though all patients received a standardized pain regimen on discharge, acute postoperative pain and narcotics use could not be assessed after the inpatient hospital period. This prevented us from evaluating differences in pain or narcotics consumption between groups in the immediate postoperative period after hospital discharge. Third, few complications occurred overall in this study population, which may have prevented us from detecting differences in complication rates between cohorts. Fourth, although VAS has been proven as a valid measure of pain, ${ }^{2,3,6}$ it does involve potential subjectivity and variability in its application, which may have limited our ability to detect small differences in pain experience between groups. Fifth, limited availability of data on factors such as preoperative depression and anxiety restricted our ability to analyze these as potential contributors to postoperative pain. Sixth, it is possible that some patients may have received an epidural steroid injection prior to surgical intervention that may have contributed to the analgesic effect observed postoperatively. Finally, limited compliance with PRO survey completion at 1- and 2-year postoperative time points prevented our assessment of long-term outcomes. However, further follow-up is ongoing to assess for complications after the use of local steroid injections. Nonetheless, further investigation is needed to evaluate long-term outcomes associated with local steroid use. Despite these limitations, this study is the first of its kind to assess the efficacy of local intraoperative steroid use specifically in MIS TLIF through a randomized controlled trial.

\section{Conclusions}

Local depomedrol application did not lead to decreases in acute postoperative pain or narcotics consumption after MIS TLIF. Additionally, local depomedrol administration was not associated with postoperative improvements in PROs. Finally, although the use of depomedrol was associated with small increases in complication rates, this was not statistically significant. The findings of this randomized trial suggest that the use of local intraoperative steroids may not provide additional benefit with regard to surgical and clinical outcomes in patients who undergo MIS TLIF. However, additional studies are needed to further assess long-term outcomes and complication risks with the use of local intraoperative steroids in lumbar fusion procedures.

\section{References}

1. Akinduro OO, Miller BA, Haussen DC, Pradilla G, Ahmad FU: Complications of intraoperative epidural steroid use in lumbar discectomy: a systematic review and meta-analysis. Neurosurg Focus 39(4):E12, 2015

2. Bijur PE, Silver W, Gallagher EJ: Reliability of the visual analog scale for measurement of acute pain. Acad Emerg Med 8:1153-1157, 2001

3. Boonstra AM, Schiphorst Preuper HR, Reneman MF, Posthumus JB, Stewart RE: Reliability and validity of the visual analogue scale for disability in patients with chronic musculoskeletal pain. Int J Rehabil Res 31:165-169, 2008

4. Copay AG, Glassman SD, Subach BR, Berven S, Schuler TC, Carreon LY: Minimum clinically important difference in lumbar spine surgery patients: a choice of methods using the Oswestry Disability Index, Medical Outcomes Study questionnaire Short Form 36, and pain scales. Spine J 8:968-974, 2008
5. Debi R, Halperin N, Mirovsky Y: Local application of steroids following lumbar discectomy. J Spinal Disord Tech 15:273-276, 2002

6. Folstein MF, Luria R: Reliability, validity, and clinical application of the Visual Analogue Mood Scale. Psychol Med 3:479-486, 1973

7. Jamjoom BA, Jamjoom AB: Efficacy of intraoperative epidural steroids in lumbar discectomy: a systematic review. BMC Musculoskelet Disord 15:146, 2014

8. Jirarattanaphochai K, Jung S, Thienthong S, Krisanaprakornkit W, Sumananont C: Peridural methylprednisolone and wound infiltration with bupivacaine for postoperative pain control after posterior lumbar spine surgery: a randomized double-blinded placebo-controlled trial. Spine (Phila Pa 1976) 32:609-617, 2007

9. Mobbs RJ, Phan K, Malham G, Seex K, Rao PJ: Lumbar interbody fusion: techniques, indications and comparison of interbody fusion options including PLIF, TLIF, MI-TLIF, OLIF/ATP, LLIF and ALIF. J Spine Surg 1:2-18, 2015

10. Modi H, Chung KJ, Yoon HS, Yoo HS, Yoo JH: Local application of low-dose Depo-Medrol is effective in reducing immediate postoperative back pain. Int Orthop 33:737-743, 2009

11. Ranguis SC, Li D, Webster AC: Perioperative epidural steroids for lumbar spine surgery in degenerative spinal disease. A review. J Neurosurg Spine 13:745-757, 2010

12. Shin SH, Hwang BW, Keum HJ, Lee SJ, Park SJ, Lee SH: Epidural steroids after a percutaneous endoscopic lumbar discectomy. Spine (Phila Pa 1976) 40:E859-E865, 2015

13. Wilson-Smith A, Chang N, Lu VM, Mobbs RJ, Fadhil M, Lloyd D, et al: Epidural steroids at closure after microdiscectomy/laminectomy for reduction of postoperative analgesia: systematic review and meta-analysis. World Neurosurg 110:e212-e221, 2018

14. Zhang Y, Yang XJ, Zeng TH, Qiu YY, Wang YT, Liang FG: A retrospective study of epidural and intravenous steroids after percutaneous endoscopic lumbar discectomy for large lumbar disc herniation. Chin J Traumatol 20:34-38, 2017

\section{Disclosures}

Dr. Singh has direct stock ownership in Avaz Surgical, LLC, and Vital 5, LLC. He is also a consultant for DePuy, Zimmer, and Stryker. He either receives royalties from or is on the board of directors for the following companies: Zimmer, Stryker, Pioneer, Lippincott Williams \& Wilkins, Thieme, Jaypee Publishing, Slack Publishing, CSRS, ISASS, AAOS, SRS, and Vertebral Column - ISASS.

\section{Author Contributions}

Conception and design: Haws, Khechen, Ahn, Bohl, Mayo, Massel. Acquisition of data: Haws, Khechen, Guntin, Cardinal. Analysis and interpretation of data: Haws, Khechen, Ahn, Bohl. Drafting the article: Haws, Khechen, Patel, Bawa. Critically revising the article: Haws, Khechen, Patel, Bawa. Reviewed submitted version of manuscript: Singh, Haws, Khechen, Patel, Bawa. Approved the final version of the manuscript on behalf of all authors: Singh. Statistical analysis: Haws, Khechen, Bohl. Administrative/technical/material support: Guntin, Cardinal. Study supervision: Singh.

\section{Supplemental Information}

\section{Previous Presentations}

Lumbar Spine Research Society, Chicago, IL, April 9-10, 2015. The International Society for the Advancement of Spine Surgery, Las Vegas, NV, April 6-8, 2016.

\section{Correspondence}

Kern Singh: Rush University Medical Center, Chicago, IL. kern. singh@rushortho.com. 